\title{
ESPECIES DE MIRIDAE (HEMIPTERA) REGISTRADAS EN ALGUNOS CULTIVOS TROPICALES EN CHANCHAMAYO Y SATIPO. JUNÍN - PERÚ
}

\section{MIRIDAE (HEMIPTERA) SPECIES RECORDED IN SOME TROPICAL CROPS AT CHANCHAMAYO AND SATIPO. JUNÍN - PERU}

\author{
Germán Arellano ${ }^{1}$ y Clorinda Vergara ${ }^{2}$
}

\begin{abstract}
Resumen
El presente estudio tuvo como objetivo identificar las especies de insectos Miridae (Hemiptera) presentes en seis cultivos tropicales en la selva del Departamento de Junín, Perú. Se considera como base las recolecciones realizadas entre los años 1983 a 1987. El muestreo se basó en colectas periódicas semanales de insectos en las plantaciones de cafeto (Coffea arabica L.), papayo (Carica papaya L.), piña (Ananas comosus var. comosus (L.) Merr., Coppens \& Leal), palto (Persea americana Mill), cítricos (Citrus aurantium L.) y plátano (Musa paradisiaca L.). Los insectos fueron colectados en campo sobre los cultivos y la hierba mediante aspiradores de succión, redes entomológicas aéreas y de golpe. Los especímenes capturados fueron remitidos al Museo de Entomología “Klaus Raven Büller” de la Universidad Nacional Agraria La Molina, para su montaje y determinación. Las determinaciones de las especies se realizaron con la colaboración de expertos del Museo Nacional de Rio de Janeiro, Brasil. En los resultados de este trabajo se establece la identificación de 55 especies de Miridae de las cuales siete fueron descritas como nuevas.
\end{abstract}

Palabras clave: Miridae, Hemiptera, fundo Génova, fundo Santa Teresa, Chanchamayo, Satipo.

\section{Abstract}

This study aimed to record and identify the species of plant bugs Miridae (Hemiptera) present in six tropical crops in the rainforest of the Department of Junín, Peru. Sampling was conducted from 1983 to 1987 in the following crops: coffee (Coffea arabica L.), papaya (Carica papaya L.), pineapple (Ananas comosus var. Comosus (L.) Merr., Coppens \& Leal), avocado (Persea americana Mill), citrus (Citrus aurantium L.) and banana (Musa paradisiaca L.). The specimens collected in the field were sent to the "Klaus Raven Büller" Museum of Entomology at the Universidad Nacional Agraria La Molina, for mounting and identification. Species determination were carried out with the collaboration of experts at the National Museum of Rio de Janeiro, Brazil. We report 55 species of plant bugs which included 7 new described species.

Key words: Miridae, Hemiptera, fundo Genova, fundo Santa Teresa, Chanchamayo, Satipo.

\section{Introducción.}

Los insectos mirídos "chinches de las plantas" (Hemiptera: Miridae) son muy diversos morfológicamente y presentan una gran plasticidad trófica, tienen una función clave en el funcionamiento de los ecosistemas naturales y agroecosistemas como fitófagos y depredadores. Con frecuencia son los más abundantes en las plantas anuales y perennes de las regiones templadas y tropicales (Wheeler, 2001).

La familia Miridae contiene más de 1300 géneros y 11130 especies descritas, es la más grande y diversa del Suborden Heteroptera. Muchos son fitófagos y pueden causar daños muy graves en los cultivos y una gran parte son depredadores que podrían tener importancia en programas de control biológico, de allí que el registro de las especies presentes en un lugar determinado es de importancia en el manejo de plagas de los cultivos. Se consideran en la Región Neotropical al menos 561 géneros y más de 3400 especies, incluidas en 7 de 8 subfamilias reconocidas en el mundo (Ferreira et al., 2015). En el Perú, Vergara \& Raven (1989) registraron en el Museo de Entomología de la Universidad Nacional Agraria la Molina, 53 especies de 32 géneros agrupadas en 6 subfamilias, como resultado de la ejecución de un proyecto cuyo objetivo fue la determinación de las especies Miridae con la colaboración del Dr. José C.M. Carvalho del Museo Nacional de Rio de Janeiro, elaborando el registro de acuerdo al orden taxonómico propuesto por Carvalho (1957, 1958a, y 1958b). Posteriormente, Carvalho (1991), establece la lista de los mirídos del Perú y describe 7 nuevas en base al material recolectado por Arellano entre 1984 y 1987, 
en 7 subfamilias. Subsiguientemente Carvalho \& Costa, 1992 describen una nueva especie en Loreto.

En este trabajo se citan las especies de Miridae (Hemiptera) recolectadas en el fundo Génova en la provincia de Chanchamayo y el fundo Santa Teresa en la provincia de Satipo, del Departamento de Junín; en los cultivos de cafeto (Coffea arabica L.), papayo (Carica papaya L.), piña (Ananas comosus var. comosus (L.) Merr., Coppens \& Leal), palto (Persea americana Mill), cítricos (Citrus aurantium L.) y plátano (Musa paradisiaca L.); en base a 503 colectas de insectos en general realizadas entre los años 1984 y 1987 que incluyeron observaciones sobre hospedadores y presencia estacional, en el marco de un programa más complejo en el que se trató de evaluar especies entomológicas dañinas y benéficas en estos cultivos (Arellano, 2001); (Lozada \& Arellano, 2008); (Arellano et al., 2015).

El objetivo del presente trabajo fue dar a conocer la relación de especies Miridae presentes en los cultivos antes mencionados, información que puede ser importante en programas de control en esta área agrícola, promisoria en el desarrollo de la Selva Central del Perú, considerando que esta familia no se ha tratado en nuevos estudios hasta la fecha.

\section{Materiales y métodos.}

Los especímenes que fueron utilizados en las determinaciones de especies fueron colectados en "dos fundos del Instituto Regional de Desarrollo Selva de la Universidad Nacional Agraria La Molina (Perú): El fundo Génova con 577 ha, en el valle de Chanchamayo en la margen izquierda del río, cerca de San Ramón entre los 940 msnm y 1200 msnm y el fundo Santa Teresa con 300 ha, situado en el valle de Río Negro a $9.5 \mathrm{~km}$ de la ciudad de Satipo sobre la Carretera Marginal, a 850 msnm” Figura 1 (Arellano, 2001; Arellano et al., 2015).

Muestreo de insectos

Como describen Arellano (2001), Lozada \& Arellano (2008) y Arellano et al. (2015), se realizaron colectas periódicas de insectos cada dos semanas en las plantaciones de café, papaya, palto, plátano, cítricos $\mathrm{y}$ en piña una semana al mes. Simultáneamente a estos muestreos también se colectó en la vegetación herbácea de estos cultivos durante los años 1984 y 1987. Los especímenes de las colectas fueron remitidos al Museo de Entomología "Klaus Raven Büller" de la Universidad Nacional Agraria La Molina para su montaje y determinación.
Se utilizó el mismo método de recolección descrito por Arellano et al. (2015), que "consistió en la captura de insectos adultos, inmaduros y puestas, mediante aspiradores tradicionales de succión, redes entomológicas aéreas y de golpe, pinzas, serrucho de podar, tijeras, etc., sobre cualquiera de las partes de las plantas cultivadas. En la hierba la colección se hizo básicamente con red de golpe, realizando batidas de ida y vuelta sobre la maleza. Las plantas que se evaluaron fueron escogidas al azar a lo largo de una línea en diagonal que cruzaba el campo, de esta forma se realizó un muestreo aleatorio simple. Las colectas duraron de 4 a 6 horas, durante el día en horas de luz y de mayor temperatura, entre las 9 y 15 horas, interdiarias. En la maleza la colecta se realizó durante 1 hora siguiendo la misma ruta una vez concluidas las observaciones de las plantas cultivadas".

De esta forma los Mirídos capturados fueron representativos de los cultivos estudiados en estos fundos. Por su parte "durante un año de 1986 hasta junio de 1987, se llevó un registro numérico de los individuos colectados por morfoespecies, de las más abundantes y comunes a los cultivos y malezas. Toda la colecta procedente del campo era seleccionada y comparada con una colección de referencia que se conservó en el laboratorio. En ella se mantenían muestras identificadas con un código" (Lozada \& Arellano, 2008). Este registro permitió establecer la frecuencia estacional de los Mirídos más abundantes. Los Mirídos generalmente pueden ser reconocidos como "insectos de tamaño mediano o pequeño, ordinariamente delicados; ocelos ausentes; rostro con 4 segmentos; cuneus habitualmente presente, embolium indiferenciado; tarsos casi invariablemente trisegmentados” (Richards \& Davies, 1984).

AREA CHANCHAMAYO - SATIPO
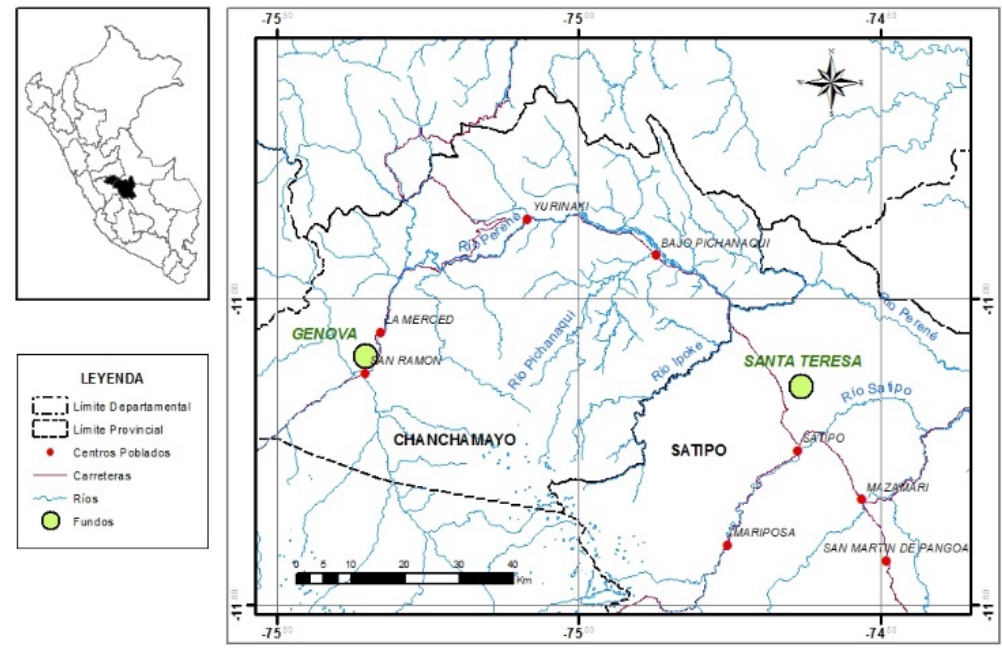

Figura 1. Ubicación de los fundos Génova en la Provincia de Chanchamayo y Santa Teresa en Provincia de Satipo (Perú). Fuente: Arellano et al., 2015. 


\section{Las determinaciones}

Se realizaron sobre el material remitido por el Museo de Entomología “Klaus Raven Büller” al Dr. J.C.M. Carvalho del Museo Nacional de Rio de Janeiro.

\section{Resultados.}

Carvalho a partir del material recolectado en los fundos Génova y Santa Teresa por Arellano entre los años 1984 y 1987, determinó 55 especies, en 7 subfamilias: Cylapinae (1), Deraeocorinae (8), Bryocorinae (14), Phylinae (6), Dicyphinae (1), Orthotylinae (5) y Mirinae (20), del material biológico depositado en el Museo de Entomología "Klaus Raven Büller” procedente de Chanchamayo y Satipo. Se incluyeron 7 nuevas especies: Antias peruana, Itacoris peruanus, Platyscytus clorindae, Perissobasis peruanus, Pliniella peruana, Pycnoderes chanchamayanus y Pycnoderes peruanus, las que fueron descritas por Carvalho en 1991 (Carvalho, 1991).

En la presente lista se consignan los nombres de las Subfamilias, especies, la clave del registro en el museo, lugares como Chanchamayo (fundo Génova) o Satipo (fundo Santa Teresa), el cultivo de recolección y en algunos casos las épocas de mayor abundancia.

Familia MIRIDAE

I. Subfamilia CYLAPINAE

1. Fulvius bisbistillatus (Stäl, 1860). (UA 1406 86) Chanchamayo, colectada sobre cafetos.

II. Subfamilia: DERAEOCORINAE

2. Annona fuscata Carvalho \& Schaffner, 1977. (UA 1974 - 85) Chanchamayo y Satipo, recolectada de mayo a diciembre sobre las plantas de piña y cafetos; también en la hierba de las plantaciones de papaya, café y cítricos.

3. Annona bimaculata (Distant, 1884). (UA 1520 86) Chanchamayo, en las herbáceas de las plantaciones de café.

4. Annona sp. (UA 1541 - 86) Chanchamayo, sobre la hierba de las plantaciones de café.

5. Antias peruana Carvalho, 1991. (UA 1357 - 86) Chanchamayo, en las plantas de cítricos.

6. Florus bolivianus Carvalho \& Gomes, 1972. (UA 2085 - 85) Chanchamayo, entre las plantas de paltos.

7. Hyaliodes inca Carvalho, 1955. (UA 1960 - 85) Chanchamayo y Satipo, sobre sobre las plantas de papayos.

8. Termatophylidea constricta Maldonado, 1970. (UA 1399 - 86) Chanchamayo, en las plantas de los paltos.

9. Florus insolitus Distant, 1884. (UA 1938 - 85) Chanchamayo, sobre la hierba de las plantaciones de café.
III. Subfamilia: BRYOCORINAE

10. Perissobasis peruanus Carvalho, 1991. (UA 2079 - 85) Chanchamayo, en los cafetos.

11. Monalocoris (?) sp. (UA 2825 - 85) Chanchamayo, sobre la hierba de las plantaciones de café.

12. Eccritotarsus chanchamaianus Carvalho \& Schaffner, 1986. (UA 1906 - 85) Chanchamayo, sin registro de hospedero.

13. Eccritotarsus cruxnigra Stäl, 1986. Figura 2a, (UA 1879 - 85) Chanchamayo y Satipo, fue abundante todo el año, en cítricos y papayos y en la hierba de las plantaciones de papaya y café.

14. Eccritotarsus machupichanus Carvalho \& Schaffner, 1986. Figura 2b, (UA 1906 - 85) Chanchamayo, fue abundante de mayo a octubre, entre las plantas de cítricos y la hierba de las plantaciones de café.

15. Parafurius sp. (UA 1949 - 87) Chanchamayo, en las plantas de cítricos, papayo y la hierba de las plantaciones de papaya.

16. Pycnoderes quadrimaculatus (Guerin-Meneville, 1857) (UA 1382 -86) Chanchamayo entre plantas de papayo.

17. Pycnoderes guaranianus Carvalho \& Gomez, 1971. (UA 1546 - 86) Chanchamayo, sobre la hierba de las plantaciones de café.

18. Pycnoderes atratus (Distant, 1884). (UA 1376 86) Figura 2c, Chanchamayo, todo el año, más abundante de enero a febrero, en las plantas de papaya, paltos, sobre la hierba de estas plantaciones, también sobre la hierba de los cítricos.

19. Pycnoderes chanchamayanus Carvalho, 1991. Figura 2d, (UA 1384 - 86), Chanchamayo, de febrero a noviembre sobre papayos, paltos $\mathrm{y}$ cafetos. También fueron registrados en la hierba de estas plantaciones y de los cítricos.

20. Pycnoderes peruanus Carvalho, 1991. (UA 1358 - 86) Chanchamayo, sobre palto, sobre la hierba de las plantaciones de café.

21. Sixeonotus brasiliensis Carvalho \& Gomes, 1971. Figura 2e, (UA 1388 - 86) Chanchamayo, en abundancia todo el año, en paltos, cafetos y la hierba de las plantaciones de cítricos, papaya y café.

22. Monalonion peruvianus Kirkaldy, 1907. (UA 1026 - 87) Chanchamayo, sobre palto. También en "pino chuncho" Schizolobium amazonicum Ducke, donde se congrega en gran número en la corteza y se alimenta en las resquebrajaduras de los tallos.

23. Macrolophus praeclarus (Distant, 1884). (UA 2089 - 85) Chanchamayo, en cítricos en el cultivo y sobre la hierba.

IV. Subfamilia PHYLINAE

24. Ellenia cuneata (Stäl, 1860). (UA 1047 - 87) Chanchamayo, colectada entre las plantas de 
cítricos y la hierba de estas plantaciones, también fue colectada sobre la hierba de las plantaciones de café.

25. Platyscytus clorindae Carvalho, 1991. (UA 1647 - 85) Chanchamayo, en agosto en la hierba de las plantaciones de café y palto.

26. Tytthus neotropicalis Carvalho, 1958. (UA 657 85) San Ramón. Sin observaciones de hospedero. Coll. G. Hospina.

27. Tytthus sp. Satipo, sobre la hierba de las plantaciones de café.

28. Sthenaridea carmelitana (Carvalho, 1948). (UA 2065 - 85), abundante todo el año entre las plantas y la hierba de las plantaciones de cítricos, también sobre plantas de cafetos y papayos.

29. Sthenaridea vulgaris (Distant, 1983). (UA 2081 85) Chanchamayo, en la hierba de los cítricos.

V. Subfamilia DICYPHINAE

30. Cyrtopeltis (Engytatus) modestus (Distant). (UA 1398 - 86). Chanchamayo sobre plantas de cítricos.

VI. Subfamilia ORTHOTYLINAE

31. Halticus bractatus (Say, 1832). (UA 2067 - 85) Chanchamayo, abundante todo el año entrelos cafetos y la hierba de las plantaciones de cítricos, palto y café.

32. Ceratocapsus sp. (UA 2005 - 85) Chanchamayo, sobre los cafetos y la hierba de las plantaciones de los cítricos.

33. Falconia veneciana Carvalho, 1990. (UA 2072 85) Chanchamayo, registrada en la hierba de las plantaciones de palto y café.

34. Itacoris peruanus Carvalho, 1991. (UA 1948 85) Chanchamayo, sobre la hierba de las plantaciones de papaya.

35. Pliniella peruana Carvalho, 1991. (UA 1360 86) Chanchamayo, en paltos.

VII. Subfamilia MIRINAE

36. Collaria oleosa (Distant, 1883). (UA 1355 - 86) Chanchamayo y Satipo, abundante de marzo a agosto en cítricos, papayos y cafetos.

37. Dolichomiris linearus Reuter, 1882. (UA 2074 85) Chanchamayo y Satipo, entre la hierba de las plantaciones de papayo y cítricos.

38. Neotropicomiris noedicus Carvalho \& Fontes, 1983. (UA 1982 - 85) Chanchamayo, sobre la hierba de las plantaciones de café.

39. Prepops vitticolis (Reuter, 1910). Chanchamayo. Sin registro de observaciones. Durojeani Coll, 1963.

40. Prepops sp. (UA 1539 - 86) Chanchamayo, sobre los cafetos.

41. Creontiades rubrinervis (Stal, 1862). (UA 1874 - 85) Chanchamayo, abundante todo el año, sobre los papayos y cítricos y la hierba de las plantaciones de estos cultivos.

42. Garganus andinus Carvalho, 1992. (UA 1992 85) Satipo y Chanchamayo, abundante desde enero a julio, sobre la hierba de las plantaciones de papaya y cítricos.

43. Garganus gracilentus (Stal, 1860). (UA 1988 85) Chanchamayo, de marzo a diciembre en los cafetos, con mayor abundancia sobre la hierba de las plantaciones de papaya, cítricos y café.

44. Garganus sp. (UA 1986 - 85) Chanchamayo, entre la hierba de las plantaciones de papaya.

45. Horciasinus signoreti (Stäl, 1859). (UA 1889 85) Chanchamayo, abundante todo el año en la hierba de las plantaciones de cítricos, café y palto.

46. Horciasinus tucumanus (Berg, 1883). (UA 1338 - 86) Chanchamayo, con mayor frecuencia de mayo diciembre, entre los papayos pero fue más abundante sobre la hierba de las plantaciones de los cítricos, palto, papayo y café.

47. Horciasoides vittatus Carvalho, 1976. (UA 2068 - 85) Chanchamayo, en la hierba de las plantaciones de cítricos.

48. Horcias pentheri Reuter, 1097. (UA 2069 - 85) Chanchamayo, en las plantaciones de palto sobre el cultivo y la hierba.

49. Perumiris machupichanus Carvalho, 1987. (UA 2015 - 85) Chanchamayo, entre la hierba de las plantaciones de café.

50. Polymerus ecuadoriensis (Carvalho \& Gomez, 1953). (UA 1994 - 85) Chanchamayo y Satipo, se presentó en abundancia de mayo a noviembre, en los cafetos, papayos y la hierba de las plantaciones de café.

51. Polymerus peruanus Carvalho \& Melendez, 1986. (UA 1925 - 85) Chanchamayo y Satipo, fue abundante todo el año, pero aumenta su densidad en octubre.

52. Polymerus testaceipes (Stäl, 1860). (UA 1932 85). Chanchamayo, en las plantaciones de papayo, sobre el cultivo y la hierba.

53. Proba vittiscutis (Stäl, 1860). (UA 1996 - 85) Chanchamayo, con mayor frecuencia de mayo a junio, en los cítricos y cafetos.

54. Taedia signata Carvalho \& Gomez, 1971. (UA 1324 - 86) Chanchamayo, entre la hierba de las plantaciones de palto.

55. Taylorilygus pallidulus (Blanchard, 1952). (UA 1950 - 85) Chanchamayo, de agosto a diciembre en las plantaciones de palto, en el cultivo y la hierba. 
a)

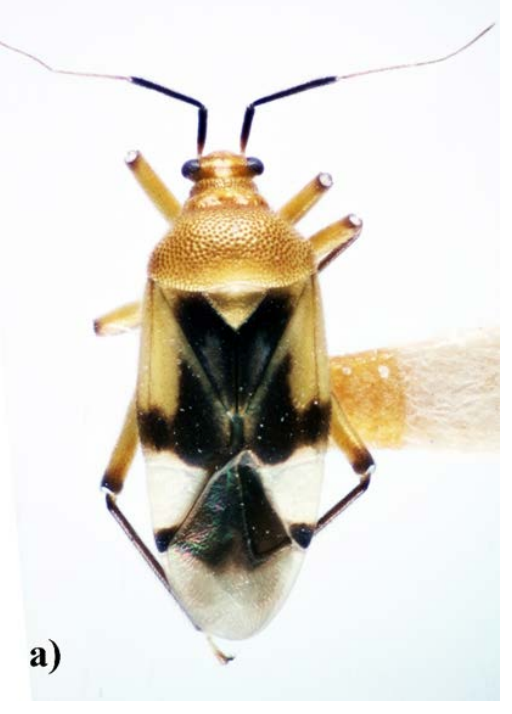

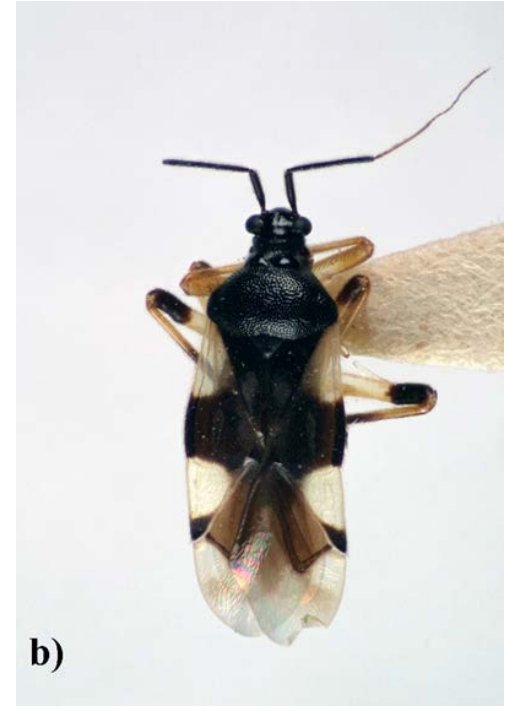

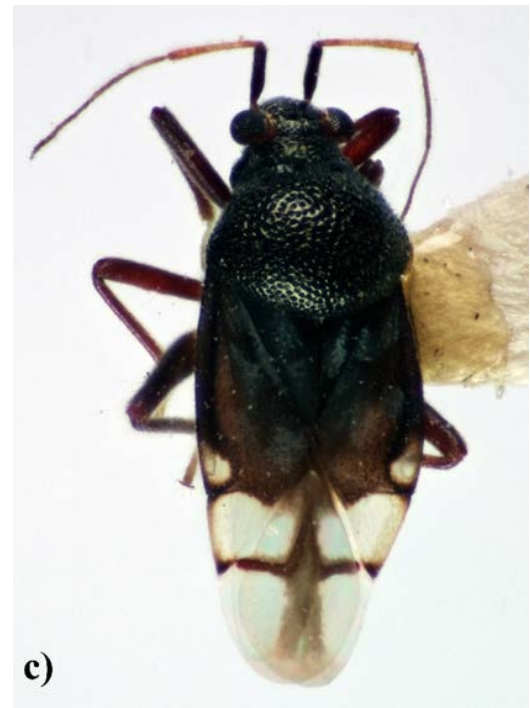

d)

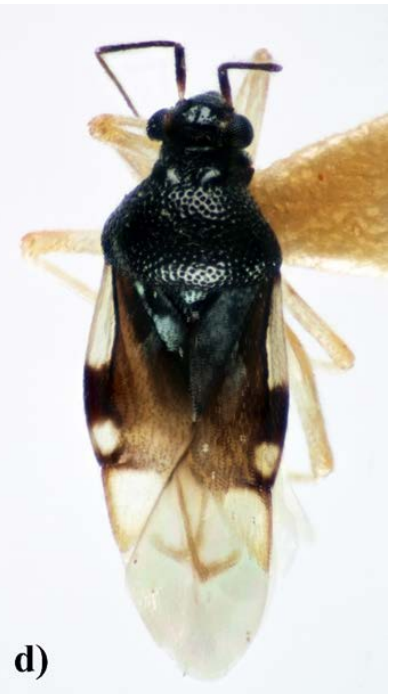

e)

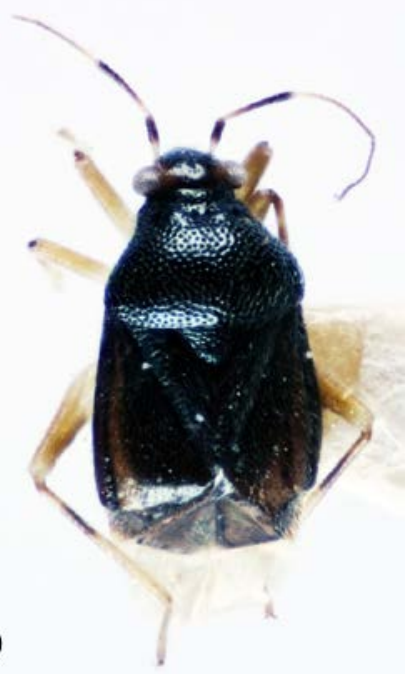

Figura 2. Fotografías tomadas por Clorinda Vergara, sobre el material que se conserva en el Museo de Entomología "Klaus Raven Büller" de la Universidad Nacional Agraria La Molina. Se presentan las especies más importantes por su abundancia y estacionalidad entre 1984-87. a) Eccritotarsus cruxnigra Stäl, 1986. Fue abundante todo el año, sobre cítricos y papayos y en la hierba de las plantaciones de papaya y café de Chanchamayo y Satipo. b) Eccritotarsus machupichanus Carvalho \& Schaffner, 1986. Fue abundante de mayo a octubre, sobre las plantas de cítricos y la hierba de las plantaciones de café, en Chanchamayo. c) Pycnoderes atratus (Distant, 1884). Se registró todo el año y en abundancia de enero a febrero, sobre las plantas de papaya, paltos, sobre la hierba de estas plantaciones, así como en la hierba de los cítricos en Chanchamayo. d) Pycnoderes chanchamayanus Carvalho, 1991. Se registró con abundancia de febrero a noviembre sobre papayos, paltos y cafetos. También fueron registrados en la hierba de estas plantaciones y de los cítricos en Chanchamayo. e) Sixeonotus brasiliensis Carvalho \& Gomes, 1971. Se observó en abundancia todo el año, sobre los paltos, cafetos y la hierba de las plantaciones de cítricos, papaya y café en Chanchamayo. 


\section{Conclusiones.}

1. En el material biológico recolectado en 503 salidas al campo de seis cultivos tradicionales en el fundo Génova (Chanchamayo) y en el fundo Santa Teresa (Satipo) son determinadas 55 especies de la familia Miridae, correspondientes a 7 subfamilias.

2. De las 55 especies son citadas como nuevas siete, las que se encuentran depositadas en el Museo de Entomología “Klaus Raven Büller” de la Universidad Nacional Agraria La Molina, Lima - Perú.

\section{Dedicatoria y agradecimiento.}

Este trabajo está dedicado a la memoria del Dr. Klaus Raven Büller gestor del proyecto inicial y a la memoria del Dr. José C.M. Carvalho por su colaboración en la determinación de las especies. Agradecimiento a todos los que de alguna manera apoyaron el trabajo de campo desde 1983 hasta 1987 en el fundo Génova, Santa Teresa y en el Museo de Entomología “Klaus Raven Büller” de la Universidad Nacional Agraria La Molina.

\section{Literatura citada.}

Arellano G. 2001. Evaluación de plagas en Café, Papayo, Piña, Palto, Plátano y Cítricos en Chanchamayo y Satipo. Tesis para optar el grado de Magister Scientiae. Escuela de Post Grado. Universidad Nacional Agraria la Molina. Lima - Perú. 194 p.

Arellano G., Vergara C. \& Bello S. 2015. Plagas entomológicas y otros artrópodos en el cultivo de la piña (Ananas comosus var. comosus (L.) Merr., Coppens \& Leal) en Chanchamayo y Satipo, Departamento de
Junín, Perú. Ecol. apl. 13(2): 175-189. DOI: 10.21704/rea.v14i1-2.94

Carvalho J.C.M. 1957. Catálogo dos Mirideos do Mundo. Parte I Subfamilias Cylapinae, Deraecorinae, Bryocorinae. Museu Nacional Rio de Janeiro, Brasil. Vol. 44.158 p.

Carvalho J.C.M. 1958a. Catálogo dos Mirideos do Mundo. Parte II Subfamilia Phylinae. Museu Nacional Rio de Janeiro, Brasil. XLV: 216 p.

Carvalho J.C.M. 1958b. Catálogo dos Mirideos do Mundo. Parte III. Subfamilia Orthotylinae. Museu Nacional Rio de Janeiro, Brasil. XLVII. 161 p.

Carvalho J.C.M. 1991. Mirideos neotropicais CCCLV: Novas espécies de Chanchamayo, Perú, com lista anterior do mesmo paîs (Hemiptera). Rev. per. Ent. 33: 87-96.

Carvalho J.C. \& Costa L. 1992. Mirideos neotropicais CCCLXXVI: sobre una espécie nova de Anomalocornis de Loreto, Perú (Hemiptera). Rev. per. Ent. 34: 29-30.

Ferreira P.S., Henry T.J. \& Coelho L.A. 2015. Plant Bugs (Miridae). In True Bugs (Heteroptera) of the Neotropics. Springer Netherlands. (pp. 237-286). DOI: 10.1007 / 978-94-017-9861-7_10

Lozada P.W. \& Arellano G.A. 2008. Lista preliminar comentada de las "cigarritas" (Insecta: Hemiptera: Cicadellidae) de Chanchamayo y Satipo, Perú. Ecol. apl. $7(1,2): 117-122$

Vergara C. \& Raven K. 1989. Miridae (Hemiptera) registrados en el Museo de Entomología de la Universidad Nacional Agraria La Molina. Rev. Per. Ent. 31:51-56. DOI: 10.21704/rea.v7i1-2.366

Richards O.W. \& Davies R.G. 1984. Tratado de Entomología Imms Volumen 2. Ediciones Omega S.A. Barcelona. 998 p.

Wheeler A.G. 2001. Biology of the plant bugs (Hemiptera: Miridae): pests, predators, opportunists. Cornell University Press. 507 p.

\footnotetext{
${ }^{1}$ Docente y Jefe del Laboratorio de Ecología de Artrópodos, Departamento de Biología, Universidad Nacional Agraria La Molina. Av. La Molina S/N, La Molina Lima - Perú. Correo electrónico: acg@lamolina.edu.pe

2 Docente y Jefe del Museo de Entomología "Klaus Raven Büller”, Universidad Nacional Agraria La Molina, Av. La Molina S/N, La Molina Lima - Perú. Correo electrónico: cvc@lamolina.edu.pe
} 